[Under review. Last draft 12.01.2009]

\title{
MAKING SENSE OF MODELING: BEYOND REPRESENTATION
}

\author{
Isabelle Peschard \\ San Francisco State University
}

\section{Abstract}

It has recently been aptly emphasized that how models are used is essential to what scientific models are. But the explanations of why and how a model is used or why a model is scientifically valuable are still merely in terms of the relation between the model and its target, just as they were before the explicit mention of uses and users. To use a model is to perform an action, and as for any action, different accounts can be given depending on the perspective that is adopted. An account of use and users in terms of relations between the model and its target is close to the poorest we could get of the role of the users and the function of models.

I will argue that models need to be regarded as elements of an epistemic space, a space of related models-of-phenomena and activities of modeling. On that view, whether a model-of- $\mathrm{X}$ is epistemically valuable or scientifically worthwhile depends on the difference it makes in this epistemic space with respect to the investigation of scientifically significant problems. I will focus on the most common way for a model to make a difference: to be used in the construction of other models.

\section{Introduction}

It has recently been aptly emphasized that how models are used is essential to what scientific models are. The reasoning runs roughly as follows: nothing is a representation in and by itself but only in virtue of being used to represent or being used as representation, and models are representations, therefore it is essential to models that they are used, used as representations. What is curious, however, is that in spite of this 
emphasis on uses and users, the explanations of why and how a model is used or why a model is epistemically valuable, scientifically worthwhile, are still merely in terms of the relation between the model and its target, just as they were before the explicit mention of uses and users.

For instance, in his inferential conception of representation, Suarez (2002) insists that 'the reference to the presence of agents and the purpose of inquiry is essential'. But the reason is that, regarding the agents, "some agent's intended uses to be in place" is required to establish and maintain the representational force of the source, and regarding the purpose, it depends on the aim of the inquiry what level of competence is required to draw inferences from the source about the target. And his assertion that "scientific representations have cognitive value because they aim to provide us with specific information regarding their target" shows that all that matters in the assessment of the cognitive value of models still lies in the relation between the model and its target. Similarly in Contessa's (2007) interpretational conception of representation, users are there to interpret the vehicle in terms of the target, and the use of the representation is surrogative reasoning about the target. Finally, in Giere's intentional conception, which does place the representational function in a larger context, the function of the agents is still restricted to 'specify[ing] which similarities are intended', and the use of the model is 'to represent physical processes in the real world' (Giere, forthcoming).

It is important to clarify the sense in which models function as representation, but it should be clear that saying that models are used as representation says very little, in fact, about their use, and that they are representations says very little about their scientific worth. Not because it is not true that models are representations, if by that one wishes to underline that a model is something meaningful, that a model is a model of something and wishes to account for the fact that models enable scientists to make some claims about what they are models of. Representation talk is not very illuminating, however, when it comes to understanding how models are constructed, how the determination of what the model should account for can be the subject of long-standing controversies, how such controversies are resolved in practice, or how the conception of the target is transformed in the course of the process of modeling. But I have discussed this aspect of models and modeling elsewhere (Peschard, 2009) and I want here to discuss still another, 
even though related, shortcoming of representation talk on models. It is that however representation is understood, focusing on representation, insofar as that would amount to simply considering the relation between the model and the phenomenon it is a model of, suffers from a dramatic lack of perspective when it comes to accounting for the use and the scientific value of models.

To use a model is to perform an action, and as for any action, different accounts can be given depending on the perspective that is adopted. To account for the use and the scientific value of a model in terms of the relation between the model and what it is a model of is a bit like explaining why I took the BART (Bay Area Rapid Transit) at 8:30am today in terms of my getting to the other side of the Bay at 9:00am. It is true that what is good about the BART is that it takes me there at the chosen time. But this understanding of the action is close to the poorest one could get. That the explanation leaves out much that is crucial becomes clear if one imagines that once arrived on the other side of the Bay the passengers cannot get out of the station. Similarly, an account of use and users in terms of relations between the model and its target is close to the poorest we could get of the role of the users and the function of models.

If the cognitive or scientific value of models were limited to the relation between models and targets, the historical importance of certain scientific models, the model of the DNA or of the atom for example, would hardly make sense, and scientific modeling as a whole would reduce to a mere accumulation of models.

I will argue that an account of the use and value of models which doesn't reduce scientific modeling to so dramatically short-sighted a kind of action needs to look beyond the relation between a model and what it is a model of.

Rather than asking about the scientific value of models, and appealing to their relation to phenomena, we should ask about the scientific value of the model-of-X as a scientific unit of use and of epistemic assessment. A crucial corollary of this modification is that models are not to be considered as situated merely somewhere along a line relating theories to phenomena. They will be regarded as elements of an epistemic space, a space of related models-of-phenomena and activities of modeling. On that view, whether a 
model-of-X is epistemically valuable or scientifically worthwhile depends on the difference it makes in this epistemic space with respect to the investigation of scientifically significant problems. I will focus on the most common way for a model to make a difference: to be used in the construction of other models, or the constructive use of a model. By being instrumental in the generation of new models, a model is an instrument of progressive transformation of an epistemic space. It opens up new possibilities of investigation.

Parts I and II will successively address the constructive use of what may be regarded as two different sorts of models, 'abstract models' and 'experimental models'. This distinction broadly corresponds the distinction made by Giere between "principled models" and "representational models", only the latter being fully specified. It is tempting to take Giere's distinction as a distinction between models being used to construct a model and models being used as a representation: principled models are models used to construct representational models, and representational models are used to represent a system. What part II will show, by looking closely at the use of some 'representational models' -- namely, fluid mechanics models of a wake and of coupled wakes -- is that one should resist the temptation. The main reason will be even in this case, the representational relation falls short of accounting either for their use or for their scientific value. What needs to be taken into account is their constructive use.

\section{Use and value of an intermediary model}

\section{1. The Landau model}

Not all models can be easily regarded as representations of something. Schrödinger's equation or Newton's laws, in and by themselves, do not enable us to calculate or infer anything specific about any particular system. The former requires the specification of an Hamiltonian and the latter that of a force function. Still, one should

think that they have some use, and that they are scientifically valuable. To distinguish, as Giere (forthcoming) or Cartwright (1999: 180) do, two sorts of models, those that are abstract and those from which specific claims about a particular system can be made, suggests that the questions of their use and scientific value will need different sorts of 
answers. On the other hand, however, that most of the models actually used by scientists are of an intermediary degree of generality suggests rather that the same sort of answer may cover the whole span and bridge the difference between the two extreme sorts of cases.

In order to clarify this issue, I will then start with such an intermediary model, which in fact constitutes a background for the models considered in the second part. This model, hereafter 'the Landau model', was proposed in 1944, by Lev Landau, Russian theoretician physicist, winner of the physics Nobel prize in 1962 for his contribution to the theory of superfluidity. The Landau model describes in the following way the growth of a perturbation in a parallel flow, when the control parameter (generally a function of the velocity of the flow) is just above the critical threshold:

$$
\mathrm{dA} / \mathrm{dt}=\left(\mathrm{a}_{\mathrm{r}}+i \mathrm{a}_{\mathrm{i}}\right) \mathrm{A}-\left(\mathrm{l}_{\mathrm{r}}+i \mathrm{l}_{\mathrm{i}}\right)|\mathrm{A}|^{2} \mathrm{~A}
$$

where $\mathrm{A}$ is the complex amplitude of a perturbation, $\left(\mathrm{a}_{\mathrm{r}}+i \mathrm{a}_{\mathrm{i}}\right)$ is the coefficient of linear growth, and $\left(1_{\mathrm{r}}+i \mathrm{l}_{\mathrm{i}}\right)$ is the coefficient of non-linear effects of saturation of the amplitude. Supposing the perturbation to be oscillatory, it describes, for a positive value of the eigenvalue $a_{r}$, the evolution of the amplitude of the perturbation as following first a linear growth and a progressive inflection of the growth rate due to the compensatory effects of the non-linear term, until the linear and non-linear contributions to the growth of the amplitude balance each other. If Re represents the control parameter of the system, $\mathrm{a}_{\mathrm{r}}$ is a linear function of $\left(\operatorname{Re}-\mathrm{Re}_{\mathrm{c}}\right)$, where $\mathrm{Re}_{\mathrm{c}}$ represents the critical value of the control parameter, the value at which the system becomes unstable and the perturbation starts growing.

The model is intermediary, between the most general and the most specified in the following sense. It is not as general as Newton's law in that the class of systems that forms the target domain is smaller: the model characterizes the dynamics of a fluid, forming a parallel flow, undergoing a change of stability resulting from the growth of a periodic perturbation, for values of the control parameter close to the critical value. But it is not as specified as a model that would have quantitative implications as to the evolution of the amplitude A for a particular experimental system. For that, the other terms of the models would have to be defined in terms of measurable physical quantities, and ascribed a particular value characterizing some particular experimental conditions. 


\section{2. Scientific value of the model}

This model was not derived from the Navier-Stokes equations, which stand as theoretical laws in fluid mechanics, nor was it based on experimental measurements. The model was not shown to account for previous measurements and was too general to allow specific inferences about a particular system. But it certainly has a historical status, it is a landmark in the study of instabilities. And this is all the more surprising given that the conception of turbulence which Landau presents in the same paper, as a motivation for the formulation of the model, is now regarded as mistaken.

In effect, according to Landau, turbulent motion consists in the superposition of an infinity of modes of different frequencies which, as the control parameter increases, become successively instable and, one after the other, start growing. This theory constituted a new approach to the longstanding and opaque problem of turbulence: "Although the turbulent motion has been extensively discussed in literature from different points of view, the very essence of this phenomenon is still lacking sufficient clearness. To the author's opinion, the problem may appear in a new light if the process of initiation of turbulence is examined thoroughly" (Landau 1944: 387). Instead of vainly trying to make sense of turbulence by looking at fully developed turbulent motion, fully unpredictable and unanalyzable, Landau proposes to look for understanding of turbulence in the process of development of turbulence. The model that he presents purportedly describes the development of the first, the most instable mode of the flow, and Landau invites the reader to do the same for the subsequent modes, each time on the basis of the new basic flow generated by the combination of previous developments.

In the $80 \mathrm{~s}$, however, it was shown that just three independent modes were sufficient to produce a chaotic evolution (Ruelle \& al. 1978). So the unpredictability characteristic of turbulent motion doesn't require an infinity of modes; Landau got the number wrong. But he was right in the method, in the way of proceeding: the new theory objected to Landau in his own terms, still giving an interpretation of the turbulent motion in terms of a combination of successive modes, and the theoretical study of turbulence is still, and more than ever, what Landau said it had to be, one that focuses on understanding the development of these modes. 
So much for the theory. As for the model, it can be read as a description of the first step of the transition towards turbulence, independently of how many steps were supposed to be needed after it. And indeed, the fate of the theory did not prevent the model from being used in the investigation of the development of the first instability in diverse types of systems. I will focus on one of these systems: the wake that develops behind a cylinder when the velocity upstream reaches a certain critical value and vortices are emitted alternatively on each side of the cylinder and carried away with the flow (see figure 1). The motion of the flow behind a cylinder (which is an idealization of the flow behind an island, a rock, a pole, the pylons of a bridge, etc.) is one of the typical problems in fluid mechanics, especially difficult to deal with because, by contrast with other typical species in the fluid mechanics garden such as the problems of the parallel flow between two planes, of the flow in a cylinder or a jet, or the flow between two concentric cylinders, it adds the complication of the obstacle, with the loss of symmetry that this implies.

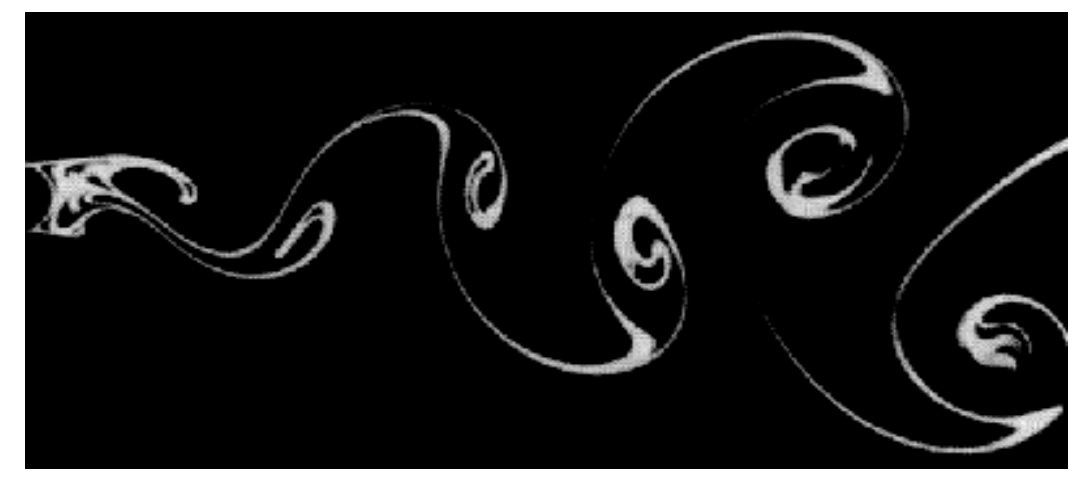

Figure 1: Wake behind a cylinder (on the left). The wake is formed by the vortices emitted on each side of the cylinder and carried away with the flow (from left to right)

The way in which the Landau model has been and is still used to investigate this and other problems, is by constituting the basic material, a template, for the construction of models, for instance models of wakes and coupled wakes. The model of the wake is obtained by specifying the order parameter and control parameter in terms of measurable 
physical quantities characterizing the experimental system of the wake ${ }^{1}$ (Mathis $\&$ al. 1984). This is how the Landau model is used. The model opens up new forms of investigation and characterization of the development of the wake in terms of the quantities and relations constituting the model. By offering a new way of thinking about the phenomenon, it made possible new questions, new challenges, and thereby pointed towards what not only could but needed to be done. Thereby it was instrumental in the development of a new, fecund and prolific research activity of modeling and experimenting. It is precisely so as to make sense of this transformative and enabling dimension of models that, Rouse argues, models should be thought of simulacra rather than representations: "simulacra ... transform the available possibilities for human action. They do so both by materially enabling some activities and obstructing others, and also by changing the situation such that some possible actions or roles lose their point, while others acquire new significance" (Rouse, 2002: 177). Not all models have such a deeply transformative effect on how to go about exploring the phenomenon. The more they have that effect, the more scientifically valuable they are.

\section{3. Use of the model}

How the Landau model is used is not different from how even more general models, like the Navier-Stokes equations or the third of Newton's laws, are used. In the same way as, say, in the model of the dynamics of a spring, the force function takes the form $\mathrm{F}=-\mathrm{kx}$, in the model the dynamics of a the wake behind a cylinder, the control parameter will take the specific form $\operatorname{Re}=\left(\mathrm{U}^{*} \mathrm{D}\right) / v$, where $\mathrm{U}$ is the upstream velocity of the flow, D the diameter of the cylinder and $v$ the viscosity. Only when U, D and $v$ are ascribed a particular value, within a certain range of values, would it be possible to make quantitative inferences from the model about the evolution of the amplitude of the perturbation that forms the wake. But in the present case, and this is not an exception, this is not the level of specification that is interesting. We do not necessarily want to think of different values of the control parameter as characterizing different systems. This would

\footnotetext{
${ }^{1}$ The amplitude of the perturbation is the amplitude, in the direction of the flow and at 5 diameters behind the cylinder of the vortices 'emitted by' the cylinder, whereas the control parameter is the Reynolds number as a function of the velocity upstream, the diameter of the cylinder and the viscosity.
} 
even be at odds with the notion of control parameter, which implies some control over one system, that is, the possibility of fixing different conditions for the same system. So if some models are to be regarded as representations of a system, that should not require that all the terms of the model take a particular value. Rather, the important difference between the Landau model and the model of the wake is that after the terms of the Landau model have been specified in terms of measurable physical quantities, these terms are quantitatively specifiable in a way that characterizes a physical system. That is, it is then possible to give values to the term representing the control parameter that correspond to some particular experimental conditions for the development of the wake.

It is not clear, if we are ready to say that of the model of the wake is a representation, why we would not say the same thing of the Landau model since they are both meaningful, both about something, even though this something differs in degree of abstraction. In addition, as I will argue in the next section, regarding models on the most specific end of the spectrum, 'being used as representation' accounts neither for their use nor for their scientific value. And even worse: the more specific the model becomes, the less illuminating its 'being used as representation' proves to be. For these two reasons, I will speak of these models that can be used to investigate specific systems, not as 'representational models' but as 'experimental models'.

\section{Use and value of experimental models}

What I call experimental models are models that are developed, that are constructed, in the context of an experimental activity. An experimental model is a model of an experimental system (in the sense of Rheinberger, 1997: 28).

\section{1. Model of a wake}

In the 90 s, the Landau model of the development of a perturbation was used to construct a model of the wake formed behind a cylinder of a very small aspect ratio (length/diameter $=\mathrm{L} / \mathrm{D}=5$ ), which will be referred to as a very 'short' cylinder. As explained before, the model for this class of systems was obtained by specifying the terms of Landau model: the control parameter is the Reynolds number, here a function of the velocity upstream, the diameter of the cylinder and the viscosity of the fluid, and the 
variables of interest are the amplitude and the frequency of the vortices emitted behind the cylinder (Peschard \& al. 1999). What characterizes a value of the aspect ratio as small is that it is far from the asymptotic limit where the value of the aspect ratio doesn't make a difference anymore to the main features of the system, such as the value of the critical Reynolds number. Because of this invariance, the results obtained in the asymptotic domain $(\mathrm{L}>50 * \mathrm{D})$ where the cylinder is regarded as infinite have a more general significance and consequently most studies are made in this domain. But the attempts to use the Landau model was confronted to some difficulties: some of the experimental results were not accounted for by the model.

On the other hand, the development of the wake in the particular conditions of a small aspect ratio could be successfully characterized in terms of the development described by the Landau model combining linear growth and non-linear saturation. The model can be decomposed into two parts, sub-models: one for the evolution of the real amplitude with the Reynolds number, the other for the evolution of the frequency. Both sub-models were shown to be in good agreement with the outcomes of measurements. But what is the scientific value of a model with such a limited domain of application?

An account of the use of models in terms of the relation between the model and its target would underline the fact that the model could then be used to predict, given a value of the Reynolds number, the value of the amplitude or the value of the shedding frequency of the system. It is true that once the model is deemed reliable, when it is regarded as accounting for the evolution of the variable, one could infer the value that the variable would take for any value of the control parameter in the range covered by the model. That may be of interest to an engineer but to the scientists, insofar as the predictions for the values of the variable agree with the measured values, it is often of little intrinsic interest what these values are. What matters is that the model accounts for the evolution of the variable. So the real question is: what is the point of having a model accounting for the evolution of this variable? The fit between the predictions of the models and the outcomes of measurements gives credential to the claim that the system in question instantiates particular mathematical relations. But whatever we learn about the system is scientifically interesting only if the system itself is scientifically interesting. 
Two main ways in which it can be scientifically interesting are
a) if it is representative of a large or significant class of system
b) if it is normally part of a larger system too complicated to be studied as a whole.

The small value of the aspect ratio characteristic of the modeled system prevents both of the above conditions to be realized. As we saw, 'small value' means well before the range of values for which the main features of the system, like the value of the critical Reynolds number, become 'universal', that is, independent of the aspect ratio. A system of high aspect ratio would be representative of a large class of systems, which is the one that is theoretically, and practically because of the large scope of application, scientifically significant. So what is the point of having a model of the wake in the small aspect ratio value type of configuration?

We need to grant scientists a vision, and see modeling as an activity embedded in a larger endeavor. The scientific value of a model may not be an immediate one but come from the role it plays in a larger project. Part of the background knowledge that will be required to really make sense of this model is that attempts to construct a model for the 'preferred' class of wakes behind an infinite cylinder had encountered puzzling difficulties. Whereas the model of the wake implies a linear relation between the value of the shedding frequency and the Reynolds number, the observations with an infinite cylinder showed a totally unexpected discontinuity in the linear evolution for a certain range of value of Re. Such a discontinuity is absent from the measurements made with a 'short' cylinder. One possible account for the difference would be that the discontinuity observed with an infinite cylinder be a mere artefact from parts of the experimental set-up that are different in the two cases (for instance the cylinder being longer the amount of irregularities in the diameter increases, the vibrations of the cylinder are more difficult to control, etc.) But the constructive of the model for a short cylinder takes place in the exploration of another possible explanation of the difference between the two cases, short and infinite cylinder, and of the discontinuity that was observed in the latter case.

It is worth noting that the above two ways for a model to be scientifically interesting take us already away from the strict relation between the model and its immediate target. But there is another way which is exemplified in this case and it takes 
us even further. It is to use the model to construct a larger system representative of a large or significant class of systems. The idea is that constructing the larger system will be a source of insight into the mechanisms at play both in its behavior and that of similar systems in the same way as constructing a machine is a way to understand how it works. . This long-term project of construction started with the model of a wake behind a short cylinder.

\section{2. Model of two coupled wakes}

The next step was to construct an experimental system by adding another identical cylinder next and parallel to the first (Peschard and Legal 1996).

The model for one wake was then used to construct a model for a system of coupled wakes. The new model is obtained on the basis of two models, one for each wake, by supplementing each model with terms representing a linear coupling between the wakes amplitude:

$$
\begin{aligned}
& \text { dt } \mathbf{A}(t)=\left(\mathbf{a r}+\text { iai) } \mathbf{A}(t)-(\operatorname{lr}+i \mathbf{l i})|\mathbf{A}(t)|^{2} \mathbf{A}(\mathbf{t})+(\mathrm{gr}+i \mathrm{gi})[(\mathrm{B}(t)-\mathrm{A}(\mathrm{t})]\right. \\
& \mathbf{d} t \mathbf{B}(t)=(\mathbf{a r}+i \mathbf{a i}) \mathbf{B}(t)-(\operatorname{lr}+i \mathbf{i i})|\mathbf{B}(t)|^{2} \mathbf{B}(\mathbf{t})+(\mathrm{gr}+i \mathrm{gi})[(\mathrm{A}(t)-\mathrm{B}(\mathrm{t})]
\end{aligned}
$$

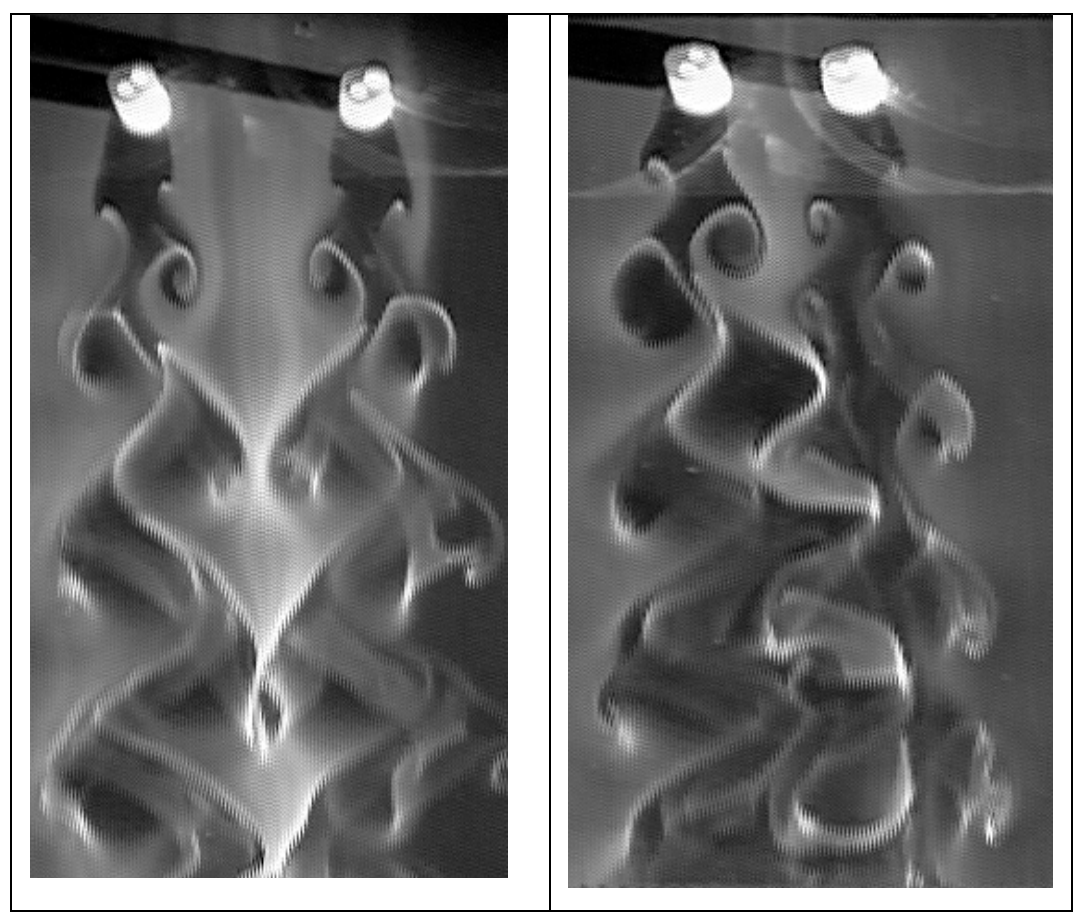

Figure 2: Two states of a system of coupled wakes developing behind cylinders placed side by side at varying distance. The two cylinder are on top of the photo, seen form above. (2a): The 
system is in a symmetric state. (2b) The system is in an asymmetric state.

Hans-Jörg Rheinberger's remark that "sufficiently stabilized epistemic things turn into the technical repertoire of the experimental arrangement" (1997:29) is particularly appropriate. What was previously the target of the experimental investigation and empirical characterization through the procedure of measurement, namely the single wake, has now become the material for the construction of a new experimental target, the system of coupled wakes.

But at the same time, what was a model under investigation, the model of a single, isolated wake, has become material for the construction of a new model, a model of the new target, the system of coupled wakes. It is to be faithful to this two dimensional activity of construction that the unit of use and assessment has to be identified as the model-of-the-wake rather than the model alone. It is the model-of-the-wake that will be deemed scientifically valuable if its use proves fruitful; and its use is intrinsically related to a new activity of modeling.

Some difficulties may seem to hamper an account of the epistemic value of a model A in terms of its use to construct a model B. First of all, it seems to lose sight of the use of models as instruments of transformation of the world. Some models are used to construct or transform, not other models, but things, bridges, planes, and foremost instruments. And here, undoubtedly their scientific value comes from the accuracy of the inferential relation they entertain with what they purport to be models of, that is, their value seems to be fully accountable in terms of the relation between the model and the phenomenon.

That's true, but those who construct these objects are engineers or technicians, and I make a distinction between the activity of the scientist and that of the technician or engineer. Models constructed in relation with an experimental system can be used by engineers or technicians to construct models of the particular system they are interested in. What makes a model valuable for the engineer is not the same as what makes a model valuable for the scientist -- fluid mechanics offers a striking example of two traditions, the scientist's and the engineer's that have developed for a long time in parallel with their 
own models disregarded by the other side: "Whereas hydrodynamicists applied advanced mathematics to flows rarely encountered by engineers, hydraulicians used simple empirical or semi-empirical formulas that defied deeper theory" (Darrigol, 2005: vi).

Another concern may be that using models to construct models looks like an aimless or at least vain activity. What's the point of replacing a view of scientific activity as mere accumulation of models, which I said earlier is what reducing our perspective on models to their relation with the purported target leads to, with one as a game of model construction? There would indeed not be much point if we didn't look further than the 2place relations between the model used to construct a new models and this model. This is why models of phenomena and their constructive use have to be located in an epistemic space. Part of what characterizes what I call, in the context of this paper, an epistemic space is a set of fundamental questions or problems ${ }^{2}$ and what makes the construction of the model scientifically valuable is the new light it casts on such problems and the new terms it offers to deal with them.

Admittedly, that raises a challenge for the model of coupled wakes: what's the point of having constructed this model? One could say, for instance, on the basis of the publication of the study on coupled wakes, that the simulation of the model makes it possible to draw a map of the possible states of the experimental system as a function of the parameters of the model. The possibility of making specific inferences about the experimental system though requires a precise coordination of the theoretical parameters of the model to measureable forms of intervention on the physical system through some systematic experimental observations. The ability to make inferences serves as regulative 'principle' in the making of the model-- but that does not mean that it is by itself epistemically valuable. Rather, it is a symptom of the achievement of the coordination

\footnotetext{
${ }^{2}$ In her study of the use of classical models of chaos to construct models of quantum chaos, Alisa Bokulich shows how this construction is a source of insight into fundamental questions like intertheoretic relations, and what makes the construction of the quantum model scientifically valuable is the new light it casts on such problems and the new terms it offers to approach them.
} 
between the model and the experimental system, with the upshot of a general understanding of the empirical meaning of the terms of the model, for instance the term of coupling, the mathematical identification of the different observable states of the system, and even a guiding tool in research pertaining to states not yet observed.

To understand how the model of coupled wakes could be expected to contribute to general problems in fluid mechanics, one more step is needed. It is the use of the model of two coupled wakes, behind short cylinders, a detail that will soon prove crucial, to construct a model of a row of similar wakes.

\section{3. Row of coupled wakes}

The new model is constructed on the basis of the previous one (Legal \& al. 1996). The term of coupling now takes into account the presence of neighbors on each sides:

$$
\mathrm{d} t \operatorname{An}(t)=\sigma \mathrm{An}(t)-l|\mathrm{An}(t)| 2 \mathrm{An}(\mathrm{t})+\mathrm{g}[\mathrm{A} \mathrm{n}+1(t)+\mathrm{An}-1(\mathrm{t})-2 \mathrm{An}(\mathrm{t})]
$$

The experimental is constituted of a row of wakes the develop behind cylinder of short aspect ratio, put side by side along the direction perpendicular to the direction of the flow (see figure 3)

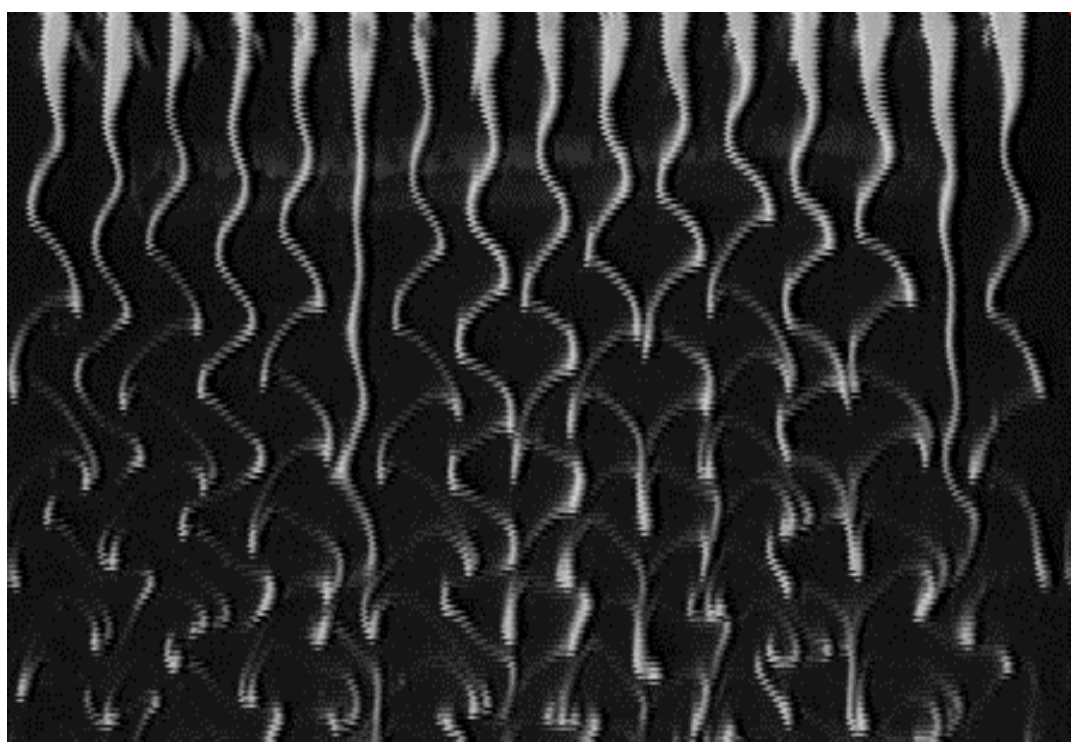

Figure 3: View from above of a row of wakes behind cylinders placed side by side in the direction perpendicular to the direction of the flow. The cylinders are on top of the photo. The fluid is flowing towards the bottom of the photo.

Here again, it is possible to give a short-sighted version of the use of the model: to 
guide, on the basis of the simulation of the model, the empirical exploration of the possible states of the system, through the mathematical identification of observed motion, or even the observation of new states of the system for a certain interval of values of the parameters which had not been explored with sufficient precision. But the 'real' motivation, the most ambitious one, lies, again, beyond the relation between the model and the system it is a model of. It locates the model within an epistemic enterprise for which it is the model-of-the-row, as epistemic unit, which is an instrument of investigation.

To understand how it plays this role we need to go back several years ago to the difficulties related to the study of the wake beyond a long cylinder. Associated with the discontinuity I mentioned was observed a phase dynamics along the axis, instead of the expected invariance by translation along the axis of the features of the shedding (Williamson, 1989). One of the hypothetical explanations proposed soon after was in terms of a continuous coupling of oscillators along the axis. The idea behind the modeling of coupled wakes, with the progressive increase of the number of wakes, was then a reaction to this proposal. By adding wakes to the system, a discrete version of the span-wise spatial dimension of the long cylinder is progressively generated with the coupling mechanism, identified with the distance between the wakes, being a controllable parameter of the system. The systematic investigation of the effect of the coupling was seen as a possible way of gaining some insight, in the continuous limit, on the role of a coupling mechanism of the phase dynamics of the wake.

Now we can see why the basic wake, element of the construction of the row of coupled wakes, had to be a wake behind a short cylinder: because in the wake that forms behind a short cylinder, there is no dynamics along the axis of the cylinder. We have a 'pure' oscillator. Hence, it is possible to regard the progressive construction of a row of coupled wakes as the generation of a spatial dimension corresponding, in the long cylinder configuration, to the spatial dimension containing the axis of the cylinder.

Now we can see also how far from the mere relation between the model and its target we may need to go in order to understand the motivation for the construction of a model and the source of the epistemic value of a model. The model of the single wake, 
behind a short cylinder, makes possible a process of construction of other models, which have to be apprehended from a perspective large enough to contain the whole project of developing an original form of elucidating or at least gaining some clarification on a system that is not among the targets of these models.

One may try to salvage the central importance of the relation of representation by objecting that the process of construction is directed at a better understanding of a particular system: the wake behind a long cylinder. Isn't the final aim to make a model of this phenomenon? Yes, it is, but only because this phenomenon is an interesting one: "Science as an ongoing practice of inquiry discounts truths that are trivial, marginal, anomalous, arcane, or otherwise 'uninteresting', in order to focus resources and attention upon others that are taken to be significantly revealing" (Rouse, 2002: 157).

Scientists rarely take the pain to explain in their scientific publication why the phenomenon they are investigating is worth investigating; 'everybody', that is, the intended readers of the article, know it. Sometimes, happily, they indulge themselves in making a few comments about it: "the flow past a cylinder, in particular, has served for nearly a century now as a model for fundamental studies of external flows. Morkovin (1964) has aptly characterized the flow past a cylinder as 'a kaleidoscope of challenging fluid phenomena' referring to the variety of fluid structures and different flow regimes that are observed in this flow" (Karniadakis \& Triantafyllou 1989). More specifically, the phase dynamics that develops along the axis of a long cylinder raises a fundamental challenge to the ability of the theory of instability in that it testifies to a three dimensional dynamics where "no three-dimensional instability of the two-dimensional basic flow is expected" (Albarede and Provansal 1995).

\section{Constructive use of models}

My emphasis on the constructive use of models and thereby on the notion of construction of models requires that I clarify the differences and similarities between this view and other approaches to the construction of models. By showing the diverse elements that were drawn on for arriving at the London model, Suarez and Cartwright 
(2008) argues that it undermines the idea of models being derived from a theory. The comparison of a theory with a toolbox suggests a process of model construction for which the theory (only) provides some pieces. Another strong case for the constructive character of modeling activity is offered by (Boumans 1999) where Boumans gives a striking illustration of the diversity of the elements that may go into the construction of a model, theoretical principles, mathematical techniques, metaphor, analogies and empirical data that needs to be accounted for by the model under construction. Whereas Suarez and Cartwright were particularly concerned with the relation between theory and models, the two main points Boumans' makes in addition is that 1) there is no general recipe for the construction of models and 2) when the model is constructed so as to account for empirical data, some empirical justification is built-in through the process of construction itself, hence blurring the distinction between procedure of discovery and procedure of justification.

The cases that I discussed could have been presented in a way that fully agree with both accounts. For instance, regarding the construction of a model of a wake, the observation of an oscillatory dynamics was among the elements of construction: the model had to account for this type of behavior. And the construction of a model of coupled wakes drew, by analogy, on other studies made with electro-dynamical oscillators to determine the form of the coupling term as linear rather than non-linear. But it is not the aim of this paper to discuss or analyze the process of construction of models. Boumans' study on the construction of model will help me to clarify the difference between this type of concern and the one that motivated this paper.

First, whereas Boumans's perspective is from the model being constructed and directed towards the elements of the construction, my perspective is from models used in the construction and directed towards the model that is to be constructed. An analysis of the way in which a model M1 is used to construct another model M2 would reveal the recourse to other elements going into the process. I am not offering such an analysis here; I am focusing on one of these elements and the fact that it is used, without details on the procedure through which it is used. Whereas it is, at least implicitly, admitted that abstract models are used to construct what I call here experimental models, the models that are then obtained are usually regarded as being used merely to represent a specific 
model. In relation to what they are model of, their target, models are they are not even used at all to represent, but rather they are used as representation, for instance to make some claims about the behavior of the target system. More importantly, I have argued that these experimental models themselves may be used, in turn, for the construction of other experimental models, that is, have a constructive use..

Second, whereas Boumans' other main concern is the built-in justification of the model that is constructed, one of my main concern is the epistemic value of models and especially the epistemic value that stems from their constructive use. The source of this epistemic value lie beyond the empirical justification of this model and beyond the use of this model to make claim about its target system; it lies beyond itself, in its use to construct other models. Making sense of modeling will then require to situate the relation between a model and its target $\mathrm{P}$ within a larger picture, a process of construction in which it is the model-of-P that is epistemically significant.

\section{Conclusion}

I have argued that in order to account for the use and the scientific value of a model, we need to look beyond the mere relation between the model and the phenomenon it is a model of. The aim of modeling activity is not to accumulate models of phenomena, as a perspective limited to the relation between models and the phenomena they are or are purported to be models of may suggest. Scientific modeling is historically motivated and future oriented, driven by the ambition to impact on the development of a domain of investigation structured around some significant questions. But what motivates modelsof-phenomena and what they make possible, the way they respond to important problems and transform the way in which these problems are to be apprehended, is not visible in the relation between models and the phenomena they are models of.

By considering the case of both abstract and experimental models, I have shown that the unit of use and assessment is not models, it is models-of-phenomena and their use and assessment pertains to an epistemic space constituted by a network of models and modeling activities. Models-of-phenomena are used as material for the construction of models and new experimental systems. The scientific value of a model of a certain phenomenon lies in its making possible new forms of investigation of phenomena 
through modeling activities building on them as basic structures or templates for new models.

\section{REFERENCES}

Albarede, P. and M. Provansal (1995) "Quasi-Periodic cylinder wakes and the GinzburgLandau model", J. Fluid Mech, 291: 191-222.

Bokulich, A. (2003) "Horizontal Models: From Bakers to Cats”, Philosophy of Science, 70: 609-627.

Boumans, M. (1999) “Built-in Justification”, in Models as Mediators, M. Morgan and M. Morrison (eds), Cambridge University Press: 66-96.

Cartwright, N. (1999) The Dappled World, A Study Of The Boundaries Of Science, Cambridge University Press.

Contessa, G. (2007) “Scientific representation, Interpretation, and Surrogative Reasoning", Philosophy of Science 74: 48-68.

Darrigol, O. (2005) Worlds of Flow, A History of Hydrodynamics from the Bernoullis to Prandtl. Oxford University Press.

Karniadakis, G. E. and G. S. Triantafyllou (1989) "Frequency selection and asymptotic states in laminar wakes", J. Fluid Mech. 199: 441.

Landau, L. (1944) "On the problem of turbulence", C. R. Acad. Sci. U.S.S.R. 44: 311-314

Legal, P.; I. Peschard, M-P. Chauve and Y. Takeda (1996) "Collective Behavior of Wakes Downstream a Row of Cylinders”, Phys. Fluids 8 (8): 2097-2106.

Giere, R. (forthcoming) “An Agent-Based Conception of Models and Scientific Representation", Synthese.

Mathis, C.; M. Provansal, and L. Boyer (1984) "The Benard- von Karman Instability: an experimental study near the threshold", J. Physique Lett. 45: 483-491.

Morkovin, M. (1964) "Flow around Circular Cylinder - a Kaleidoscope of Challenging Fluid Phenomena”, ASME Symposium on Fully Separated Flows: 102-118.

Peschard, I. (2009) "Modeling and Experimenting”, Presentation at Models and Simulation 3, University of Virginia, Charlottesville, March 6-8. 
Peschard, I. and P. Legal (1996) "Coupled Wakes of Cylinders", Physical Review Letters, 77 (15): 3122-3125.

Peschard, I. and P. Legal (1999) "On the Spatio-Temporal Structure of Cylinder Wakes", Experiments in Fluids, 26: 188-196.

Rheinberger, H-J (1997) Towards a History of Epistemic Things. Stanford University Press.

Rouse, J. (2002) How Scientific Practices Matter. Reclaiming Philosophical Naturalism. The University of Chicago Press.

Suarez, M. (2004) “An inferential conception of representation”, Philosophy of Science $71,767-779$

Suarez, M. and N. Cartwright (2008) "Theories: Tools vs. Models", Studies in the History and Philosophy of Modern Physics, 39: 62-81.

Williamson, C.H.K. (1989) "Oblique and parallel modes of vortex shedding in the wake of a circular cylinder at low Reynolds number" J. Fluid Mechanics 206: 579-627. 